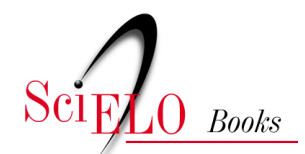

\title{
III - Soberanía alimentaria, sustentabilidad y cambio climático La Ley Orgánica de Régimen de Soberanía Alimentaria en el Marco de los Objetivos de Desarrollo Sostenible y la Agroecología
}

\author{
Narcisa Requelme
}

\section{SciELO Books / SciELO Livros / SciELO Libros}

REQUELME, N. La Ley Orgánica de Régimen de Soberanía Alimentaria en el Marco de los Objetivos de Desarrollo Sostenible y la Agroecología. In: ESTEBAN, D., ARTACKER, T., and LIZANO, R., coords. Cambio climático, biodiversidad y sistemas agroalimentarios: avances y retos a 10 años de la Ley Orgánica del Régimen de la Soberanía Alimentaria en Ecuador [online]. Quito: Editorial Abya-Yala, 2020, pp. 203218. ISBN: 978-9978-10-466-8. http://doi.org/10.7476/9789978105689.0012.

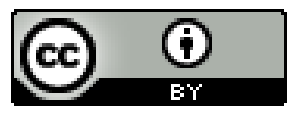

All the contents of this work, except where otherwise noted, is licensed under a Creative Commons Attribution 4.0 International license.

Todo o conteúdo deste trabalho, exceto quando houver ressalva, é publicado sob a licença Creative Commons Atribição 4.0.

Todo el contenido de esta obra, excepto donde se indique lo contrario, está bajo licencia de la licencia Creative Commons Reconocimento 4.0. 


\section{La Ley Orgánica de Régimen de Soberanía Alimentaria en el Marco de los Objetivos de Desarrollo Sostenible y la Agroecología}

\section{Introducción}

El problema de la alimentación en el mundo no es nuevo, desde los movimientos sociales y los Estados se han propuesto acciones para solucionarlo. Una de ellas fue el reconocimiento de la alimentación como un derecho. Para ello, los países están en la obligación de respetar, proteger y efectivizar el derecho, es decir, buscar los mecanismos para que todas las personas en especial las subalimentadas, tengan acceso, regular, permanente y libre, de manera directa o la compra, a una alimentación cuantitativa y cualitativamente adecuada y suficiente, en correspondencia a sus tradiciones culturales, que les garantice una vida psíquica y física, individual y colectiva libre de angustias, satisfactoria y digna (Golay \& Özden, s/f). Para muchas personas del mundo desarrollado este derecho se da por sentado,

1 Docente investigadora de la Universidad Politécnica Salesiana- Ecuador, Magister y candidata a $\mathrm{PhD}$ en Planificación de proyectos de Desarrollo rural y gestión sostenible en la Universidad Politécnica de Madrid. 
pues se considera que los alimentos son abundantes, desconociendo que esa abundancia se basa en estructuras frágiles de un sistema alimentario y agrícola altamente dañino y controlado, no por quienes producen los alimentos, sino por quienes ostentan el poder a diferentes niveles (Vía Campesina, 2018).

Los Estados para su gestión, tienen como marco de acción las políticas y normas, entre ellas las relacionadas a las leyes sobre seguridad y soberanía alimentaria que en América Latina son varias, reflejándose la importancia que se ha dado al tema en la región (Vivero, 2010). Estas leyes, incluyendo la Ley Orgánica del Régimen de la Soberanía Alimentaria (LORSA), fueron analizada por Cofre, Yagüe y Moncayo (2015), en las dimensiones que abordan sobre la seguridad alimentaria: i) acceso; ii) disponibilidad; iii) utilización biológica; y, iv) estabilidad; y en la dimensión de la soberanía alimentaria: i) producción; ii) diversidad cultural; iii) recursos naturales; $y$, iv) equidad/género, concluyendo que la LORSA, se relaciona con el acceso y la utilización biológica (art. 7,8, 26), enfoca como derecho la diversidad cultural (art. 31 y 32), los recursos naturales (art. 5, 6 y 8) y la producción (art. 12, 13, 16 y 17), es decir, tiene un enfoque centrado en la soberanía alimentaria que va más allá de la seguridad alimentaria.

Quienes se veían amenazados por procesos que van consolidando el control de los sistemas agroalimentarios, propusieron la Soberanía alimentaria como una apuesta para la resolución de las crisis que enfrenta el planeta y la humanidad. Se la considera como un derecho de los pueblos y Estados a definir su política agraria y alimentaria, sin dumping frente a países terceros (Vía Campesina, 2003). Se trata de un cambio sistemático en el que las personas tienen el control directo y democrático de los elementos importantes para la producción y la decisión de qué consumir sin perder de vista los beneficios para las generaciones futuras (Vía Campesina, 2018).

En el Ecuador los movimientos sociales plantearon la LORSA como política normativa infraconstitucional (Córdova \& Zapatta, 
2017) que materializa lo establecido en la Constitución ecuatoriana. La ley tiene detrás procesos organizativos y de lucha de diversos colectivos quienes al ver afectados sus derechos a la producción y alimentación, la plantearon para asumir la problemática de acceso a los factores de la producción, de la comercialización y abastecimiento de alimentos sanos e inocuos, pero también como una propuesta de cambio de los modelos de producción bajo el enfoque de la Agroecología. Se propuso además un esquema de participación social activo a través del Sistema Nacional de Soberanía Alimentaria (SISAN) y la Conferencia Plurinacional de Soberanía Alimentaria (COPISA).

Luego de largos procesos de debate y negociación política se obtuvo una ley que tiene como objetivo establecer los mecanismos mediante los cuales el Estado garantiza a las personas, comunidades y pueblos la autosuficiencia de alimentos sanos, nutritivos y culturalmente apropiados de forma permanente. Fue necesario establecer una normativa integral para propender a la construcción de políticas agroalimentarias, esto le dio el carácter de orgánica, por lo que, la LORSA contiene a otras conexas como la de recursos hídricos, usos y aprovechamiento del agua (art 5), ley de tierras rurales y territorios ancestrales (art. 6), la de agrobiodiversidad, semillas y fomento de la agricultura sustentable (art. 8), de sanidad agropecuaria (art. 24 y 25) y de economía popular y solidaria (art. 18, 21 y 23).

Por otro lado, a nivel mundial, frente a un amplio abanico de problemas globales, los países acordaron trabajar bajo una Agenda hasta el 2030, donde se establecieron acciones clave para construir un futuro común, donde nadie se quede atrás. La Agenda 2030 es un plan de acción en favor de las personas, el planeta y la prosperidad, pone la igualdad y dignidad de las personas en el centro y llama a cambiar nuestro estilo de desarrollo respetando el medio ambiente (ONU, 2015). La LORSA se promulgó antes de la Agenda 2030, tiene el mérito de haberse anticipado a varias de las metas que se plantean en los objetivos de desarrollo sostenible (ODS) y fue propuesta desde la lógica del cambio de paradigma hacia el Buen Vivir. 
El objetivo en este artículo fue analizar el contenido de la ley en concordancia con el ODS 2 de hambre cero, el de producción y consumo sostenible (ODS 12), y la Agroecología como aporte al establecimiento de nuevos modelos de producción y consumo sostenibles planteados en la Agenda 2030 que es un compromiso del Estado y que permitirá dar cuenta de la intervención de varios de los aspectos agroproductivos y sociales de las zonas rurales que originaron el surgimiento de la LORSA. Se eligió el ODS 2 por considerarlo como uno de los más estratégicos, pues está directamente ligado al respeto, la protección y la garantía del ejercicio del derecho a la alimentación. Se seleccionó el ODS 12 porque se relaciona con la producción sostenible, base del sistema alimentario, y el consumo sostenible. Se buscó analizar la relación con el concepto de Agroecología porque es la ciencia que plantea las bases ecológicas para la conservación de la biodiversidad en la agricultura y apunta al restablecimiento del balance ecológico de los agroecosistemas para lograr una producción sustentable y sostenible, además de ser una apuesta política de los movimientos sociales en Ecuador y Latinoamérica. Se planteó la pregunta ¿Cómo la LORSA es congruente con las metas de los ODS 2 y 12 , y el logro de la soberanía alimentaria con enfoque agroecológico hacia nuevos modelos de producción y consumo?

Metodológicamente se realizó el análisis a través de una búsqueda de concordancias de palabras clave en el texto de la ley con las metas de los ODS 2 y 12. Posterior a ello, se realizó una lectura crítica para identificar el articulado congruente con cada una de las metas. También se analizó la ley en su relación con el concepto de Agroecología bajo los criterios económicos: equidad, viabilidad económica, uso de recursos locales, producción estable; los criterios ambientales: biodiversidad, función económica; estabilidad productiva; y, los criterios sociales: desarrollo rural integrado, satisfacción de necesidades, autosuficiencia alimentaria. Las fuentes de consulta fueron documentos oficiales de acceso público y la información generada a través de entrevistas semiestructuradas con funcionarios. Esta investigación se enmarca en los estudios de doctorado de la autora sobre 
la "Sustentabilidad en Sistemas de producción lechera de agricultura familiar altoandina y buen vivir rural en Ecuador" que está desarrollando en la Universidad Politécnica de Madrid.

\section{Breve contexto}

La Agenda 2030 plantea que los países logren la seguridad alimentaria para ello involucrarán a actores públicos y privados, entre ellos a las grandes empresas que inciden en la gobernanza de los sistemas agroalimentarios desde dentro o fuera del Estado colocando en primer plano sus intereses económicos sin importar los efectos ambientales negativos que generan. Promover la seguridad alimentaria y no la soberanía alimentaria es una cuestión que se apega al carácter flexivo de los ODS, pues se deja en libertad a los Estados para que implementen políticas acordes a consideraciones locales y particulares. Cuando los países adoptan el enfoque de la seguridad alimentaria, despojan al derecho a la alimentación, de todo el contenido político de consolidación de propuestas locales propias y diferentes al modelo hegemónico de abastecimiento, distribución y consumo de alimentos.

Otra observación a la Agenda 2030, ha sido su complejidad no sólo por el número de objetivos, sino por considerarse como ambiciosos, universales y absolutos, esto generado por la variación local que pueden establecer cada país en su implementación. Se cuestiona la naturaleza contradictoria de muchas metas donde los logros de crecimiento económico son incompatibles con las metas ambientales (ODS 8, meta 8.4) y la sostenibilidad (Liverman, 2018), término que está mencionado en 10 de los ODS, pero sin una definición clara de lo que es y lo que implica. Plantear el crecimiento económico como objetivo también denota que se consolida el modelo económico vigente sin buscarse otro tipo de economías alternativas donde el principio no sea la acumulación y donde el desarrollo no sea asumido como un fin. La producción sostenible sugerida en la Agenda 2030, está condicionada a la variación de las condiciones climáticas 
originadas por causas antropocéntricas, y en los ODS, solamente las metas $1.5,2.4,11 \mathrm{~b}, 13.1$ y $14 \mathrm{~b}$ están relacionadas a la resiliencia al cambio climático. De igual forma la agenda se enmarca en el paradigma de la cientificidad y la tecnología como únicas fuentes de conocimiento, sugiriendo ligeramente el rescate y puesta en valor de los saberes locales a los que se los incorpora sólo en la meta 2.5. A pesar de estas observaciones, la Agenda 2030, no deja de constituirse en una oportunidad para articular esfuerzos por mitigar la compleja situación de hambre, pobreza, contaminación y demás condiciones de vulnerabilidad de la naturaleza y los seres humanos (ONU, 2015), los Estados tienen que dar cuenta del avance del logro de los ODS, que es un proceso altamente político por la naturaleza de las decisiones que se tienen que tomar y donde es imperante revisar las políticas e impulsar una profunda reorganización de las pautas de consumo y producción (Gómez, 2017).

En la Agenda 2030, se abordan todos los derechos humanos, entre ellos el de la salud, interrelacionándolo con el derecho a un ambiente que la promueva y proteja, para que no sólo se la aborde desde el enfoque biomédico y atención médica (Hawkes \& Buse, 2016), sino, prestando atención a factores determinantes como es la alimentación. ${ }^{2}$ En el ejercicio del derecho a la alimentación e inclusión, está la disminución de la malnutrición infantil, dada por la ingesta inadecuada de alimentos o por enfermedades, que son factores directamente relacionados con la inseguridad alimentaria en los hogares, las prácticas de atención y alimentación, entorno doméstico y servicios públicos de salud inadecuados (FAO, OPS, WFP \& UNICEF, 2018).

En el Ecuador, la FAO como organismo de apoyo a los Estados, ha acompañado la elaboración del marco de cooperación para el logro de los ODS. En él se establecen tres prioridades en torno a la

2 Brasil, resalta por su política de hambre cero con una estructura social que vigila el cumplimiento de la política de manera activa y proactiva desde este amplio concepto de salud (Feldenheimer da Silva, Recine, Jhons, Da Silva, Araujo \& Faerstein, 2018). 
alimentación: i) seguridad alimentaria y nutricional, ii) agricultura y desarrollo rural; y iii) manejo de recursos naturales y gestión de riesgo. Se contempla la creación de condiciones políticas, sociales e institucionales que contribuyan a la erradicación del hambre e incentiven la dotación y el consumo de dietas saludables. Se han establecido como objetivos: a) ayudar a eliminar el hambre, la inseguridad alimentaria y la malnutrición; y, b) fomentar sistemas agrícolas y alimentarios más inclusivos y eficientes, todo esto ligado a las metas de los ODS 2, 5 y 12. Bajo estos lineamientos se han diseñado y se están ejecutando varios programas y proyectos de responsabilidad de varios ministerios.

\section{La LORSA y los ODS 2 y 12}

La ley guarda gran congruencia con el ODS 2. Se destaca el planteamiento de seguro alimentario (art 19) y subsidios agroalimentarios (art 21) orientados a garantizar el acceso y disponibilidad de alimentos protegiendo la producción (meta 2.1). Si comparamos el articulado entre la meta 2.3 y 2.4 se nota un énfasis en la productividad e ingresos (meta 2.3) y en menor medida el aseguramiento de la sostenibilidad de los sistemas de producción y la aplicación de prácticas agrícolas resilientes (meta 2.4). En la LORSA, se deja expresamente declarado que se promoverá la reconversión sustentable de procesos productivos convencionales a modelos agroecológicos para el aseguramiento de la soberanía alimentaria. La LORSA se adelantó a los ODS al contemplar en su articulado principios para el fomento de la producción tales como: la inclusión económica, social y territorial; principios comunitarios: solidaridad y la equidad, así como aquellos que son parte de la propuesta de soberanía alimentaria: interculturalidad, protección de saberes ancestrales, equidad de género, sustentabilidad, entre otros, que buscan consolidar una posición ideológica y política en el discurso, en la norma y que se espera se concrete en la práctica de las intervenciones del Estado.

Nótese también un énfasis de la normativa con relación a la meta 2 a referida a los medios a través de los cuales se puede alcanzar los 
ODS, en este caso, el incremento de inversiones en infraestructura rural, especialmente de riego, para investigación y desarrollo tecnológico en varios aspectos de la producción, sostenibilidad, uso y aprovechamiento eficiente de los recursos productivos. A diferencia de los ODS que plantea la investigación desde una perspectiva cientificista con leve incorporación de los saberes locales, desde la LORSA se enfatiza el desarrollo de la investigación aplicada y participativa y un sistema de extensión basado en el diálogo de saberes valorando el conocimiento local de hombres y mujeres (art. 9), lo cual consolida la soberanía alimentaria y desarma dependencias externas, es este caso, de conocimiento.

\section{Tabla 1}

\section{Relación del articulado de la LORSA con el ODS 2}

\begin{tabular}{|c|c|c|}
\hline $\mathbf{N}^{\circ}$ & Meta & Articulado \\
\hline Meta 2.1. & $\begin{array}{l}\text { Poner fin al hambre y asegurar el acceso de todas } \\
\text { las personas a una alimentación sana, nutritiva y } \\
\text { suficiente durante todo el año. }\end{array}$ & $1,12,13,18,20,22,29,34$ \\
\hline Meta 2.2. & $\begin{array}{l}\text { Poner fin a todas las formas de malnutrición y } \\
\text { abordar las necesidades de nutrición de las ado- } \\
\text { lescentes, las mujeres embarazadas y lactantes y } \\
\text { las personas de edad. }\end{array}$ & $1,3,24,27,28,31$ \\
\hline Meta 2.3. & $\begin{array}{l}\text { Duplicar la productividad agrícola y los ingresos } \\
\text { de los productores con acceso a la tierra, insu- } \\
\text { mos, a los conocimientos, los servicios financie- } \\
\text { ros y mercados }\end{array}$ & $\begin{array}{l}1,2,3,4,5,6,7,8,9,13,14,16 \\
17,18,19,20,25,26,34\end{array}$ \\
\hline Meta 2.4. & $\begin{array}{l}\text { Asegurar la sostenibilidad de los sistemas de } \\
\text { producción de alimentos y aplicar prácticas } \\
\text { agrícolas resilientes que aumentan la productivi- } \\
\text { dad y producción, contribuyan al mantenimien- } \\
\text { to de los ecosistemas, fortalezcan la capacidad } \\
\text { de adaptación al cambio climático, y mejoren } \\
\text { progresivamente la calidad del suelo. }\end{array}$ & $2,3,4,6,7,13,14,16,17,19,26$ \\
\hline Meta 2.5. & $\begin{array}{l}\text { Mantener la diversidad genética de semillas, } \\
\text { plantas, y animales, y promover el acceso a los } \\
\text { beneficios por utilización de los recursos genéti- } \\
\text { cos y los conocimientos tradicionales. }\end{array}$ & $2,7,8,9,12,26,34$ \\
\hline Meta 2.a & $\begin{array}{l}\text { Aumentar las inversiones en infraestructura } \\
\text { rural, investigación y servicios de extensión agrí- } \\
\text { cola, desarrollo tecnológico y bancos de genes de } \\
\text { plantas y ganado a fin de mejorar la capacidad } \\
\text { de producción agropecuaria. }\end{array}$ & $\begin{array}{l}2,4,5,6,7,8,9,10,11,13,23 \\
25,26,34\end{array}$ \\
\hline
\end{tabular}




\begin{tabular}{|l|l|l|}
\hline Meta 2.b & $\begin{array}{l}\text { Corregir y prevenir las restricciones y distorsio- } \\
\text { nes comerciales en los mercados agropecuarios, } \\
\text { mediante la eliminación paralela de todas las } \\
\text { medidas de exportación con efectos equivalentes. }\end{array}$ & $14,19,20,23,34$ \\
\hline Meta 2.c & $\begin{array}{l}\text { Adoptar medidas para asegurar el buen funcio- } \\
\text { namiento de los mercados de productos básicos } \\
\text { alimentarios y sus derivados y facilitar el acceso } \\
\text { oportuno a la información sobre mercados. }\end{array}$ & $14,19,20,23$ \\
\hline
\end{tabular}

Elaboración: La autora

Fuente: La investigación

Existe congruencia de la ley con el ODS 12, meta 12.1, donde se plantea que el Estado debe incentivar el consumo de alimentos nutritivos de origen agroecológico, pero no se plantea un articulado específico sobre información al consumidor de lo que es el consumo sostenible, el saber comer y la comida soberana como una acción política que va más allá del consumo de alimentos (Giraldo \& McCune 2019) y donde se incluye el entorno saludable, especialmente en las zonas rurales (ONU, 2012). No se evidencia articulado orientado a la educación del consumidor en general, pero sí a nivel de los programas de estudios de educación básica para fomentar el consumo equilibrado de alimentos sanos y nutritivos (art. 28).

La ley contempla la incorporación de mecanismos para promover, determinar y certificar la calidad y el contenido nutricional de los alimentos (art. 24 y 25) y si bien es cierto que se ha avanzado en el uso del semáforo para alertar sobre el contenido de grasa, azúcar y sal, aún se necesitan políticas intersectoriales y medidas fiscales sobre los alimentos procesados y bebidas azucaradas que disminuyan su consumo (Díaz et al., 2017). El "ecoetiquetado" como lo mencionan Castro y Castillo (2018) es una forma de fomentar el consumo sostenible y abordar la preocupante situación de la venta de productos en supermercados donde el 79\% tienen información nutricional, pero sólo el 21\% coloca información sobre el depósito de desechos, un $17 \%$ incorpora símbolos del tipo de plástico de envase, $6 \%$ usa el logo de reciclaje, $4 \%$ el logo de certificación ambiental y el $2 \%$ 
señalan que tienen un impacto ambiental reducido, ante lo cual, el consumidor no tiene todos los elementos para decidir al momento de adquirir el mejor alimento con bajo impacto ambiental.

La LORSA al igual que los ODS, se enfoca en el rescate y cuidado de la biodiversidad como base del patrón alimentario y de riqueza propia del país (art. 3,6,7,8,13,14,16) con lo cual se asegura una alimentación sana, nutritiva (art. 3 y 27) y soberana (art 1). Existe un limitado articulado para lograr la gestión y el uso eficiente de los recursos naturales pues se puntualiza el acceso a ellos (meta 12.2) y se desarrolla ampliamente las propuestas en las leyes conexas correspondientes. Se notan vacíos en lo relacionado a la reducción del desperdicio de alimentos en la cadena de producción y consumo (meta 12.3), lo cual significa no asumir directamente el problema de la ineficiencia energética de los alimentos en todo su ciclo. De igual manera, no se norma la gestión y reducción de la generación de desechos provenientes de las actividades productivas agroempresariales (meta 12.5), cuestión preocupante, mucho más si éstos son de origen químico y pueden ser fuente de contaminación especialmente del agua.

Existe otro vacío de articulado de la ley que aliente a las empresas a adoptar prácticas sostenibles y que además generen información sobre la sostenibilidad (meta 12.6). Si el cumplimiento de los ODS es tarea de todos, las empresas son actores clave para incidir en cambios importantes sobre los esquemas de producción, distribución y consumo de alimentos, el Estado debe presionar para que contribuyan a ello. El abordaje de la meta 12.7, es débil en la ley, siendo este un pilar importante de apoyo a la producción de agricultura familiar campesina y mucho más aquella de origen agroecológico. Existen experiencias exitosas en otros países donde se han establecido leyes específicas y reglamentación clara para la compra pública sostenible en beneficio de los pequeños productores y en apoyo al desarrollo de zonas rurales (Giraldo \& McCune 2019). Finalmente, no se establece normativa para subsidios ineficientes por el consumo de combustibles fósiles utilizados básicamente en la producción de alimentos de manera agroempresarial. 
Tabla 2

Relación del articulado de la LORSA con el ODS 12

\begin{tabular}{|c|c|c|}
\hline Meta 12.1. & $\begin{array}{l}\text { Aplicar el Marco Decenal de Programas sobre moda- } \\
\text { lidades de consumo y producción sostenible. }\end{array}$ & $\begin{array}{l}1,2,3,13,24,25,27,28 \\
30,31,34\end{array}$ \\
\hline Meta 12.2. & $\begin{array}{l}\text { Lograr la gestión sostenible y el uso eficiente de los } \\
\text { recursos naturales. }\end{array}$ & $3,5,6,14,34$ \\
\hline Meta 12.3. & $\begin{array}{l}\text { Reducir a la mitad, el desperdicio de alimentos en } \\
\text { la venta al por menor y a nivel de los consumidores } \\
\text { y reducir las pérdidas en las cadenas de producción } \\
\text { y suministro. }\end{array}$ & \\
\hline Meta 12.4. & $\begin{array}{l}\text { Lograr la gestión ecológicamente racional de los } \\
\text { productos químicos y los desechos a fin de minimizar } \\
\text { sus efectos adversos en la salud humana y el me- } \\
\text { dio ambiente. }\end{array}$ & 13 \\
\hline Meta 12.5. & $\begin{array}{l}\text { Reducir la generación de desechos mediante ac- } \\
\text { tividades de prevención, reducción, reciclado } \\
\text { y reutilización. }\end{array}$ & \\
\hline Meta 12.6. & $\begin{array}{l}\text { Alertar a las empresas a que adopten prácticas soste- } \\
\text { nibles e incorporen información sobre la sostenibili- } \\
\text { dad en su ciclo de presentación de informes. }\end{array}$ & \\
\hline Meta 12.7. & $\begin{array}{l}\text { Promover prácticas de adquisición pública que sean } \\
\text { sostenibles, de conformidad con las políticas y priori- } \\
\text { dades nacionales. }\end{array}$ & $14,20,34$ \\
\hline Meta 12.8. & $\begin{array}{l}\text { Asegurar que las personas tengan la información y los } \\
\text { conocimientos pertinentes para el desarrollo sosteni- } \\
\text { ble y los estilos de vida en armonía con la naturaleza. }\end{array}$ & 3,14 \\
\hline Meta 12.a & $\begin{array}{l}\text { Ayudar a los países en desarrollo a fortalecer su capa- } \\
\text { cidad científica y tecnológica para avanzar hacia mo- } \\
\text { dalidades de consumo y producción más sostenibles. }\end{array}$ & $2,9,10,11,25,26,33,34$ \\
\hline Meta 12.b & $\begin{array}{l}\text { Elaborar y aplicar instrumentos para vigilar los } \\
\text { efectos en el desarrollo sostenible, a fin de lograr } \\
\text { un turismo sostenible que cree puestos de trabajo y } \\
\text { promueva la cultura y los productos locales. }\end{array}$ & $6,17,34$ \\
\hline Meta 12.c & $\begin{array}{l}\text { Racionalizar los subsidios ineficientes a los com- } \\
\text { bustibles fósiles, mediante la reestructuración de los } \\
\text { sistemas tributarios y la eliminación gradual de los } \\
\text { subsidios perjudiciales. }\end{array}$ & \\
\hline
\end{tabular}

Elaboración: La autora

Fuente: La investigación 
Las leyes deben contar con una normativa más específica que las operacionalice, la LORSA al no contar con un reglamento, se dificulta su concreción en la práctica, tampoco se deja establecido el proceso de evaluación a fin de establecer el nivel de cumplimiento de parte de las instituciones garantistas del derecho a la alimentación, pero también de parte de los impulsores a fin de determinar su utilidad, aplicabilidad y posibles modificaciones.

\section{La LORSA y la agroecología}

Respecto a la agroecología, la LORSA tiene un énfasis sobre el criterio de producción estable y biodiversidad, más, si en la Agroecología, todos los criterios tienen igual importancia (Altieri \& Nicholls, 2000), se evidencia un débil abordaje sobre los criterios de función económica, satisfacción de necesidades locales y autosuficiencia alimentaria, con lo cual no se estaría apuntalando procesos de desarrollo local en base a la producción agroecológica. Por otro lado, los cambios que puedan asumir los productores, unido a la implementación de las leyes para el cambio del modelo alternativo, va de la mano con la mejora de las condiciones de infraestructura productiva, de salud, educación y vivienda en el campo que debe proveer el Estado.

Tabla 3

Relación del articulado de las leyes con el concepto de agroecología

\begin{tabular}{|l|l|l|}
\hline \multirow{4}{*}{ Dimensión } & \multicolumn{1}{|c|}{ Criterios } & \multicolumn{1}{c|}{ Articulado } \\
\hline \multirow{4}{*}{ Económica } & Equidad, viabilidad económica & $1,4,6,12,14,33$ \\
\cline { 2 - 3 } & Uso de los recursos locales & $2,3,6,9,13,16$ \\
\cline { 2 - 3 } & Producción estable & $1,2,3,13,14,16,17,19,20,23,28$ \\
\hline \multirow{4}{*}{ Ambiental } & Biodiversidad & $1,2,6,7,8,9,13,16,26,34$ \\
\cline { 2 - 3 } & Función económica & 6,12 \\
\cline { 2 - 3 } & Estabilidad productiva & $6,13,21$ \\
\hline \multirow{3}{*}{ Social } & Desarrollo rural integrado & $2,9,12,13,15,17$ \\
\cline { 2 - 3 } & Satisfacción de necesidades locales & 21 \\
\cline { 2 - 3 } & Autosuficiencia alimentaria & 19,20 \\
\hline
\end{tabular}

Elaboración: La autora

Fuente: La investigación 


\section{Conclusiones}

La relación de la LORSA con los ODS analizados, evidencia la necesidad de precisar normativas que permitan la interacción de ministerios de diferentes sectores responsables de implementar las leyes conexas para que los procesos sean eficientes, los resultados se logren en el menor tiempo y se articulen los esfuerzos para consolidar el régimen de soberanía alimentaria y procesos de desarrollo local endógenos orientados al Buen Vivir. Pero esta articulación debe darse desde una visión

La articulación de la LORSA con los ODS debe basarse en vacíos identificados en esta investigación de manera que se reporten los avances en relación a todas las metas, pero además que se dé cuenta de los avances en soberanía alimentaria y el cambio de modelos de producción bajo un enfoque agroecológico. Desde los movimientos sociales se continúa fomentando la soberanía alimentaria a través de la LORSA y las otras leyes conexas, lo cual no es contrario a lograr los ODS, sino más bien, permite recalcar que como país se tiene un paradigma alternativo al Desarrollo, donde la soberanía alimentaria es un eje que permitirá el ejercicio pleno del derecho a la alimentación y la inclusión.

La aplicación del Marco decenal para la producción y el consumo sostenible (ONU, 2012) se refleja en la LORSA, sin embargo, los temas pendientes para cambiar los patrones de consumo existentes, pasan por un trabajo de educación para la sostenibilidad en varios ámbitos de la sociedad como son los entornos escolares a través de propuestas como la Agroecología escolar (Pita \& Acevedo, 2018), los espacios comunitarios asegurando el relevo generacional y la trasmisión de conocimientos y saberes locales (Fabron \& Castro, 2019), los hogares, y espacios públicos y laborales. Conlleva también a implementar estrategias de educación a ciudadanos y consumidores sobre dietas y estilos de vida saludables y concienciación pública para visibilizar los efectos económicos, sociales y ambientales negativos de estos esquemas de producción y consumo insostenibles y no saludables a nivel personal, familiar y colectivo que representan un gasto para el Estado. 
La seguridad alimentaria y la soberanía alimentaria enfrentan desafíos grandes que demandan acciones de adaptación al cambio climático y estrategias de resiliencia que deben ser impulsadas por el Estado y la sociedad civil organizada en pleno ejercicio de sus derechos, para ello, la agroecología asumida como un enfoque que permite el cambio de modelos de producción, a más de estar incorporada en otras leyes conexas, debe transversalizarse en todas las acciones del Estado o proponerse como lo tiene Cuba a nivel de política de Estado, no condicionada por la línea ideológica de quienes estén al frente del Estado (Giraldo \& McCune, 2019), sino como una apuesta nacional implementada a diferentes niveles de gobierno para tener mayor impacto. Para ello, es también urgente iniciar no una, sino varias transiciones a diferentes escalas, niveles y dimensiones, lo que Tittonell (2019), denomina "múltiples transiciones". Los métodos y principios agroecológicos sólo serán posibles de masificarse cuando la alimentación y la agricultura sean cuestiones políticas en torno a las cuales la sociedad forme un nuevo consenso sobre una visión política y un proyecto transformador que va más allá de la esfera productiva (Rosset, Val, Pinheiro \& McCune, 2019).

La evaluación de la LORSA, así como de las leyes conexas es una tarea pendiente de parte de quienes las incentivaron y promovieron, pues no se cierra el ciclo de la política. Para ello, es necesario activar el sistema de participación establecido en la ley (SISAN y COPISA) a fin de que puedan cumplir con las funciones establecidas en la ley. Para la reforma de la Ley se deben tomar en cuenta los vacíos de ésta con los ODS, pero desde la perspectiva nacional, donde se enfatice en el concepto de soberanía alimentaria, desde la perspectiva de una apuesta de la agroecología como ciencia, práctica y movimiento político ideológico que permita revertir la gobernanza del sistema agroalimentario, cambiando las relaciones capitalistas de propiedad social de los recursos productivos y estratégicos del Estado como base para la sostenibilidad (Tilzey, 2019). 


\section{Bibliografía}

Altieri, M., \& Nicholls, C. (2000). Agroecología. Teoría y práctica para una agricultura sustentable. 1era Edición. Serie Textos básicos para la formación ambiental. Programa de la Naciones Unidas para el Medio Ambiente. Red de formación ambiental para América Latina y el Caribe. México.

Castro Salcedo, J., \& Castillo Castro, R. (2018). Ecoetiquetado en el Ecuador: cambio de paradigma. Bistua: Revista de la Facultad de Ciencias Básicas, 16(2), 79-87.

Cofre, E., Yagüe, J.L., \& Moncayo, M. (2015). Seguridad y soberanía alimentaria: análisis comparativo de las leyes en siete países de América Latina. Revista española de Estudios Agrosociales y Pesqueros, 242, (49-88).

Córdova Alarcón, L. C., \& y Zapatta Carpio, A. (2017). Soberanía alimentaria en Ecuador y Bolivia. Políticas y normativa. Revista Ciencias Sociales, 1(39).

Díaz A.A., Veliz, P.M., Rivas-Mariño, G., Vance Mafla, C., Martínez Altamirano, L.M., \& Vaca Jones, C. (2017). Etiquetado de alimentos en Ecuador: implementación, resultados y acciones pendientes. Revista Panamericana de Salud Pública, 41, e54.

Fabron, G., \& Castro, M. (2019) Agricultura a pequeña escala en tierras altas y tierras bajas. Estudio comparativo entre la Quebrada de Humahuaca y el partido de Florencio Varela. Mundo Agrario, 20(43), e109. https://doi.org/10.24215/15155994e109

FAO, OPS, WFP \& UNICEF (2018). "Panorama de la seguridad alimentaria nutricional en América Latina y el Caribe. Desigualdad y sistemas alimentarios". Santiago.

Feldenheimer da Silva, A. C., Recine, E., Johns, P., da Silva Gomes, F., de Araújo Ferraz, M., \& Faerstein, E. (2018). History and challenges of Brazilian social movements for the achievement of the right to adequate food. Global Public Health. https://doi.org/10.1080/1744 1692.2018.1439516

Giraldo, O. F., \& McCune, N. (2019). Can the state take agroecology to scale? Public policy expereines in agroecological territorialisation from Latin América. Agroecology and Sustainable Food Systems, 43(7-8), 785-809.

Golay, C., \& Özden, M. (2005). El derecho a la alimentación. Un derecho humano fundamental estipulado por la ONU y reconocido por los 
tratados regionales y por numerosas constituciones nacionales. Centre Europe-Tiers Monde (CETIM). Ginebra.

Gómez Gil, C. (2017). Objetivos de Desarrollo Sostenible (ODS): una revisión crítica. Papeles de relaciones ecosociales y cambio global, 140, 107-118.

Hawkes, S., \& y Buse, K. (2016). Searching for the Right to Health in the sustainable Development Agenda. Comment on 'Rights Language in the Sustainable Development Agenda: Has Right to Health Discourse and Norms Shaped Healt Goals?. International Journal of Health Policy and Management, 5(5), 337-339.

Liverman, D. (2018). Geographic perspectives on development goals: Constructive engagements and critical perspectives on the MDGs and SDGs. Dialogues in Human Geography, 8(2) 168-185.

ONU (2012). Marco decenal de programas sobre modalidades de consumo y producción sostenibles. Río+20. Conferencia de las Naciones Unidas sobre el Desarrollo Sostenible. Río de Janeiro.

(2015). Transformar nuestro mundo: La Agenda 2030 para el Desarrollo Sostenible. Resolución aprobada por la Asamblea General del 25 de septiembre del 2015.

Pita Paredes, M. J., \& Acevedo Osorio, Á. (2018). Contribuciones de la agroecología escolar a la soberanía alimentaria: caso Fundación Viracocha. Praxis \& Saber. Revista de Investigación y Pedagogía, 10(22), 195-220.

Rosset, P., Val, V., Pinheiro, L., \& McCune, N. (2019). Agroecology and La Vía Campesina II. Peasant agroecology schools and the formation of a sociohistorical and political subject. Agroecology and Sustainable Food Systems, 43(7-8), 895-914.

Tilzey, M. (2019). Authoritarian populism and neo-extractivism in Bolivia y Ecuador: the unresolved agrarian question and the prospects for food sovereignty as counter-hegemony. The Journal of Peasant studies, 46(3), 626-652.

Tittonell, P. (2019). Las transiciones agroecológicas: múltiples escalas, niveles y desafíos. Revista FCA UNCUYO, 51(1), 231-246.

Vía Campesina (2003). ¿Qué es la soberanía alimentaria? Recuperado de: https://bit.ly/3bMkUwP

(2018). Soberanía alimentaria ya. Una guía para la soberanía alimentaria. European Coordination Vía Campesina. Recuperado de: https://bit.ly/3249TTa

Vivero Pol, J. L. (2010). El hambre y las leyes del hombre. El derecho a la alimentación y leyes de seguridad alimentaria en América Latina en el 2010. Recuperado de: https://bit.ly/2SAPeCS 